\title{
MODELOS DE REGRESSÃO LINEARES PARA ESTIMATIVA DE PRODUTIVIDADE DA SOJA NO OESTE DO PARANÁ, UTILIZANDO DADOS ESPECTRAIS
}

\author{
ERIVELTO MERCANTE ${ }^{1}$, RUBENS A. C. LAMPARELLI ${ }^{2}$, MIGUEL A. URIBE-OPAZO ${ }^{3}$, \\ JANSLE V. ROCHA ${ }^{4}$
}

\begin{abstract}
RESUMO: O trabalho teve o objetivo de avaliar modelos lineares de regressão entre resposta espectral e produtividade em soja, na escala regional. Para isso, foram monitorados 36 municípios do oeste do Paraná, utilizando cinco imagens do satélite Landsat 5/TM da safra de 2004/2005. Foram realizados os procedimentos de transformação radiométrica e correção atmosférica nas imagens, determinando valores físicos das refletâncias aparente e de superfície. Posteriormente, foram calculados os índices de vegetação NDVI e GVI, os quais, por meio de regressões lineares simples e múltiplas, compararam-se com as produtividades oficiais dos municípios, obtidas das estatísticas IBGE. Aplicou-se também uma análise de diagnóstico, para detectar pontos influentes e de colinearidade. Os resultados mostraram que a média dos valores de NDVI e GVI de todas as imagens foi mais bem relacionada com a produtividade do que para cada data separadamente. $\mathrm{O}$ uso de regressões múltiplas com os dois índices, em todas as datas, propiciou melhores resultados de relação com a produtividade.
\end{abstract}

PALAVRAS-CHAVE: índices de vegetação, dados orbitais, cultura anual, monitoramento.

\section{LINEAR REGRESSION MODELS TO SOYBEAN YIELD ESTIMATE IN THE WEST REGION OF THE STATE OF PARANÁ, BRAZIL, USING SPECTRAL DATA}

\begin{abstract}
The main objective of this work was to evaluate the linear regression between spectral response and soybean yield in regional scale. In this study were monitored 36 municipalities from the west region of the states of Parana using five images of Landsat 5/TM during 2004/05 season. The spectral response was converted in physical values, apparent and surface reflectances, by radiometric transformation and atmospheric corrections and both used to calculate NDVI and GVI vegetation indices. Those ones were compared by multiple and simple regression with government official yield values (IBGE). Diagnostic processing method to identify influents values or collinearity was applied to the data too. The results showed that the mean surface reflectance value from all images was more correlated with yield than individual dates. Further, the multiple regressions using all dates and both vegetation indices gave better results than simple regression.
\end{abstract}

KEYWORDS: vegetation indexes, orbital data, annual crop, monitoring.

\section{INTRODUÇÃO}

A soja é uma das oleaginosas mais semeadas no Brasil e assume posição estratégica como uma das principais commodities da pauta de exportações. Saber onde está plantada e quanto vai produzir determina mudanças nas áreas social, econômica e ambiental. A cultura foi, até há alguns anos, a mais plantada nos estados do Sul do Brasil, atualmente ela está migrando para os estados do Centro-Oeste e Nordeste, apesar de o Paraná e Rio Grande do Sul ainda manterem as posições de segundo e terceiro maiores produtores dessa cultura, com 12 milhões e 10 milhões de toneladas, respectivamente (IBGE, 2008a). É importante, nesse cenário, que sejam desenvolvidos modelos de

\footnotetext{
${ }^{1}$ Eng $^{\mathrm{o}}$ Agrícola, Professor Adjunto, CCET/UNIOESTE, Cascavel - PR, Fone: (0XX45) 3220.3228, eriveltomercante@ yahoo.com.br.

${ }^{2}$ Eng ${ }^{\circ}$ Agrícola, Pesquisador, CEPAGRI/UNICAMP, Campinas - SP.

${ }^{3}$ Estatístico, Prof. Associado, CCET/UNIOESTE, Cascavel - PR.

${ }^{4}$ Eng ${ }^{\circ}$ Agrícola, Prof. Livre-docente, FEAGRI/UNICAMP, Campinas - SP.

Recebido pelo Conselho Editorial em: 26-3-2009

Aprovado pelo Conselho Editorial em: 3-2-2010
} 
estimativa de safras, que auxiliem neste processo. O estudo de modelos para estimativa de safras já vem sendo realizados desde a década de 1980, primeiro explorando dados no formato nível de cinza (NC) e, algumas vezes, com dados auxiliares (BOATWRIGHT \& WHITEFIELD, 1986). Em outras ocasiões, utilizando índices de vegetação (GROTEN, 1993). Os modelos inicialmente se baseavam em uma data, geralmente na qual havia maior relação da biomassa com a produtividade (RUDORFF \& BATISTA, 1990), mas, recentemente, o foco passou a ser no monitoramento e acompanhamento ao longo do ciclo da cultura (DORAISWAMY et al., 2004; KASTENS et al., 2005; MELO et al., 2008). Esses modelos utilizam o sensoriamento remoto de alta resolução temporal e baixa resolução espacial, levando em conta, em alguns casos, parâmetros espectrais e agrometeorológicos.

Alguns modelos alcançam bons resultados, como o de MELO et al. (2008), mas baseiam-se em dados agrometeorológicos, que nem sempre são fáceis de obter. Modelos mais simples, considerando apenas a resposta espectral da cultura, podem ser uma boa alternativa como indicam os trabalhos de SIMÕES et al. (2005) e ALMEIDA et al. (2006), apesar de terem sido realizados para cana-de-açúcar. Assim, este trabalho tem o objetivo de avaliar modelos lineares de regressão entre resposta espectral e produtividade em soja, na escala regional.

\section{MATERIAL E MÉTODOS}

A cena do satélite Landsat 5/TM (Thematic Mapper) órbita/ponto 223/77 utilizada abrange a maioria dos municípios da região oeste do Estado do Paraná. Para este estudo, foram escolhidos 36 municípios que têm grande expressão em termos de produção, na região de maior produção de soja, dentro da cena 223/77 monitorada. Os municípios estudados foram: Anahy, Araruna, Assis, Boa Esperança, Braganey, Cafelândia, Campina Lagoa, Campo Bonito, Campo Mourão, Cascavel, Corbélia, Corumbataí, Farol, Formosa Oeste, Goioere, Iguatu, Iracema Oeste, Janiópolis, Jesuítas, Juranda, Luziana, Mamborê, Maripá, Nova Aurora, Nova Cantu, Ouro Verde, Palotina, Peabiru, Quarto Centenário, Rancho Alegre do Oeste, Santa Tereza, São Pedro, Toledo, Tuneiras, Tupãssi e Ubiratã.

O clima da região estudada apresenta-se como temperado mesotérmico e superúmido, tipo climático Cfa - Köeppen, com temperaturas moderadas, chuvas bem distribuídas e verão quente. Nos meses de inverno, a média de temperatura é inferior a $16^{\circ} \mathrm{C}$ e, nos meses de verão, as máximas superam $30^{\circ} \mathrm{C}$, com temperatura média anual de $21^{\circ} \mathrm{C}$. A região está sujeita a geadas, embora elas não sejam frequentes. Na região em estudo, encontram-se principalmente os solos do tipo Latossolo e Nitossolo Vermelho e o Neossolo Litólico (IBGE, 2008b).

Foram utilizadas informações cartográficas dos limites municipais das regiões do Estado do Paraná abrangente da cena do Landsat 5/TM utilizada, obtidas do IBGE - rede municipal da versão 2001. As malhas estaduais e municipais foram adquiridas em formato DXF e imagens do satélite Landsat 5/TM, adquiridas no Instituto Nacional de Pesquisas Espaciais (INPE), com o nível de correção denominado L1g e calibração relativa, utilizando-se do coeficiente CCRS-CPF (Canadá Centre for Remote Sensing - Calibration Parameter File) para todas as imagens, garantindo assim os mesmos procedimentos de calibração relativa para as imagens.

$\mathrm{O}$ monitoramento da cultura foi realizado por meio de imagens referentes às fases de emergência, florescimento e pico vegetativo da cultura, especificamente nas datas 23-11-2004, 9-2-2004, 25-12-2004, 26-1-2005 e 11-2-2005, portanto safra de 2004/2005. Os dados de produtividade da cultura foram coletados, na escala regional, junto à Secretaria de Agricultura e Abastecimento do Paraná - SEAB, para 36 municípios estudados (SEAB/DERAL, 2005). Os dados de produtividade foram utilizados para a realização de correlações e regressões com os dados dos índices de vegetação oriundos das imagens de satélites.

Na Figura 1, pode ser visualizado o fluxograma das etapas utilizadas no processamento das imagens Landsat 5/TM, desde a transformação radiométrica, e correção atmosférica até a geração dos índices de vegetação NDVI e GVI (MERCANTE, 2007). A transformação radiométrica foi 
realizada para converter o sinal que é captado pelo sensor, em radiância ou refletância dos alvos. Este procedimento foi realizado segundo CHANDER \& MARKHAM (2003).

As imagens foram corrigidas utilizando o sistema de correção atmosférica de imagens de satélite denominado SCORADIS (ZULLO JÚNIOR, 1994), Para a execução do algoritmo de correção do SCORADIS, nas imagens do satélite Landsat 5/TM, foram necessários os seguintes dados de entrada: 1) localização geográfica (latitude e longitude); 2) data e hora de passagem do satélite; 3) tipo e quantidade de aerossóis presentes; 4) modelo atmosférico dos componentes gasosos, principalmente vapor d'água e ozônio.

Os parâmetros atmosféricos necessários para o procedimento de correção atmosférica (espessura óptica dos aerossóis, conteúdo de vapor d'água em $\mathrm{g} \mathrm{cm}^{-2}$ e espessura da camada de ozônio em $\mathrm{cm} \mathrm{atm}^{-1}$ ) foram obtidos por meio de imagens geradas pelo sensor orbital MODIS (MOD04_L2: MODIS Level 2 Aerosol over Land and Ocean Product) a bordo da plataforma Terra.

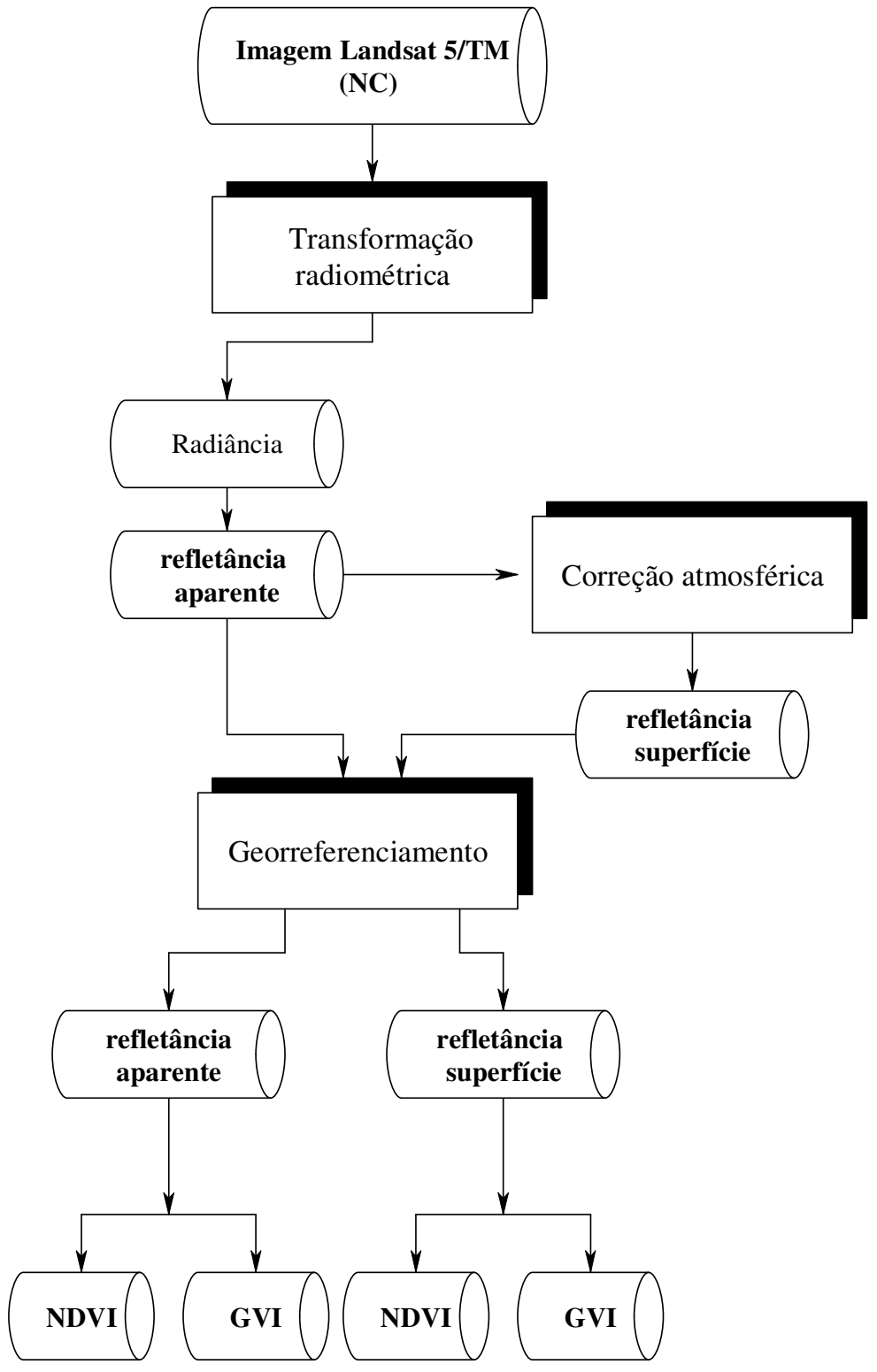

FIGURA 1. Fluxograma do processamento das imagens Landsat 5/TM. Flowchart of the processing of Landsat 5/TM images.

O horário de passagem do MODIS e, portanto, da aquisição dos atributos, foi próxima à da passagem do Landsat 5/TM, fator essencial, uma vez que as condições atmosféricas têm grande variação no tempo. 
Os valores dos atributos atmosféricos obtidos no sensor MODIS para a área do estudo estão descritos na Tabela 1. Esses valores equivalem à média aritmética de todos os pixels válidos que se encontravam dentro da área abrangente da cena 223/077 do satélite Landsat 5/TM. Posteriormente, as imagens foram georreferenciadas de acordo com os procedimentos descritos em CRÓSTA (1993).

TABELA 1. Parâmetros atmosféricos obtidos por meio das imagens MODIS. Atmospheric parameters obtained by means of MODIS images.

\begin{tabular}{cccc}
\hline Passagem Satélite & $\begin{array}{c}\text { Espessura Óptica dos } \\
\text { Aerossóis }\end{array}$ & Vapor d'Água $\left(\mathrm{g} \mathrm{cm}^{-2}\right)$ & Ozônio $\left(\mathrm{cm} \mathrm{atm}^{-1}\right)$ \\
\hline $23-11-2004$ & 0,057 & 3,229 & 0,283 \\
$9-12-2004$ & 0,163 & 2,802 & 0,277 \\
$25-12-2004$ & 0,086 & 2,500 & 0,272 \\
$26-1-2005$ & 0,071 & 3,803 & 0,262 \\
$11-2-2005$ & 0,125 & 2,233 & 0,406 \\
\hline
\end{tabular}

No intuito de caracterizar a resposta espectral da biomassa da cultura da soja e a sua relação com a produtividade final, foram geradas imagens referentes aos índices de vegetação extraídos de dentro das áreas que foram caracterizadas como ocupadas pela cultura. Para isto, utilizou-se uma máscara da soja, realizada a partir das imagens do Landsat 5/TM e por meio do processamento digital de imagens. Primeiramente, foram feitas as classificações das áreas ocupadas com soja para cada uma das cinco imagens ao longo da safra de 2004/2005; ao final, a máscara de soja foi gerada pela sobreposição das cinco imagens classificadas, conforme MERCANTE et al. (2006).

Os índices de vegetação utilizados no trabalho foram de duas abordagens distintas: uma baseada na razão de bandas, que é o índice NDVI - Normalized Difference Vegetation Index, o qual se resume na relação entre as medidas espectrais das bandas do infravermelho próximo e do vermelho (ROUSE et al., 1973) e outra na transformação espectral, Tasseled Cap (Brightness, Greenness e Wetness) proposta originalmente por KAUTH \& THOMAS (1976). Foi utilizada a componente "Greenness", denominada índice GVI - Green Vegetation Index, que usa as seis bandas não termais dos satélites Landsat 5/TM ou Landsat 7/ETM ${ }^{+}$, sendo atribuídos pesos diferentes para cada uma delas através de transformações lineares. Os índices NDVI e GVI, para este trabalho, foram calculados a partir dos valores em Refletância aparente (topo da atmosfera - ref. apar.) e com os valores de Refletância na superfície (correção atmosférica - ref. sup.). O problema da presença, quase constante, de nuvens foi tratado pela identificação e retirado dos pixels contaminados para cada safra, por meio da classificação dos pixels que possuíam valores abaixo dos valores reais, ou seja, pertencentes à cultura da soja, no período de seu desenvolvimento, dando origem assim a uma máscara para cada data. Os pixels contaminados foram eliminados de suas respectivas imagens.

Para os 36 municípios monitorados, a extração dos dados espectrais do NDVI e GVI foi calculada pela média municipal da resposta espectral contida nos pixels classificados como Soja (máscara de soja de cada município estudado). As análises estatísticas exploratórias foram aplicadas aos valores de produtividade da soja, aos valores dos índices de vegetação NDVI e GVI de todas as imagens Landsat 5/TM. Foram testadas as diferenças das médias para os valores de cada índice de vegetação NDVI e GVI municipal, calculados a partir das refletâncias aparente e de superfície em cada uma das datas de imagem, conforme teste de $\mathrm{t}$, de comparação de médias. A normalidade dos dados foi realizada por meio do teste de Anderson-Darling. Ambos os testes foram aplicados com significância de $5 \%$.

Partindo do pressuposto acima, foram realizadas análises de correlação e regressão entre dados de produtividade (variável predita) e dados espectrais (variável preditora) oriundos dos índices de vegetação (NDVI e GVI). Essas análises tiveram o objetivo de determinar a porcentagem de explicação dos índices de vegetação (NDVI e GVI) sobre a produtividade da 
cultura da soja, assim como verificar se houve diferença na explicação da produtividade da soja por meio dos índices de vegetação calculados a partir do REF. apar. e a partir do REF. sup.

Em modelos de regressão linear, podem existir observações (dados) que têm uma grande influência sobre os resultados de ajuste do modelo. Devido a isto, são frequentemente utilizados alguns procedimentos para detectar a influência potencial de um ou mais dados sobre o ajuste do modelo. A tais procedimentos são denominados de técnicas ou medidas de diagnóstico e propõem-se a analisar a: presença de pontos muito influentes (leverage); colinearidade entre colunas da matriz $X$ (caso de regressão linear múltipla); valores discrepantes nas observações e afastamentos sérios das suposições para construção de modelos (GALEA et al., 2003). Tais testes foram realizados no software SPSS 13.0 (SPSS 2001). Esses critérios foram implantados em função do número de pontos e do número de variáveis, utilizados em cada um dos modelos lineares.

Foi testada a existência ou não de multicolinearidade entre os índices utilizados conjuntamente (NDVI e GVI) por meio do teste de Durbin-Watson, a 5\%, realizados através do MINITAB 14 (MINITAB, 2003).

\section{RESULTADOS E DISCUSSÃO}

Na Tabela 2, apresentam-se os resultados das análises de estatística dos índices de vegetação médios NDVI e GVI para os 36 municípios.

TABELA 2. Estatística descritiva dos dados dos índices médios de vegetação NDVI e GVI nos 36 municípios estudados, extraídos das imagens oriundas dos tratamentos: refletância aparente e refletância da superfície, para a safra de 2004/2005. Descriptive statistical data of average vegetation indices NDVI and GVI in the 36 cities studied, extracted from the images coming from the treatments: apparent reflectance and surface reflectance for the $2004 / 2005$ harvest.

\begin{tabular}{|c|c|c|c|c|c|c|c|c|}
\hline Data & IV & Tratamento & Média & $\begin{array}{l}\text { Desvio- } \\
\text {-Padrão }\end{array}$ & C.V. $(\%)$ & Mín. & Máx. & p-valor \\
\hline \multirow{4}{*}{ 23-11-2004 } & \multirow{2}{*}{ NDVI } & ref. apar. & $0,30 \mathrm{~b}$ & 0,04 & 13,24 & 0,23 & 0,38 & 0,01 \\
\hline & & ref. sup. & $0,34 \mathrm{a}$ & 0,04 & 11,55 & 0,26 & 0,43 & $0,05^{*}$ \\
\hline & \multirow{2}{*}{ GVI } & ref. apar. & $0,05 b$ & 0,01 & 18,63 & 0,03 & 0,08 & 0,01 \\
\hline & & ref. sup. & $0,07 \mathrm{a}$ & 0,01 & 12,67 & 0,05 & 0,11 & $0,05 *$ \\
\hline \multirow{4}{*}{$9-12-2004$} & \multirow{2}{*}{ NDVI } & ref. apar. & $0,43 \mathrm{~b}$ & 0,07 & 15,97 & 0,33 & 0,60 & 0,02 \\
\hline & & ref. sup. & $0,49 \mathrm{a}$ & 0,08 & 16,44 & 0,36 & 0,65 & 0,03 \\
\hline & \multirow{2}{*}{ GVI } & ref. apar. & $0,11 \mathrm{~b}$ & 0,03 & 25,15 & 0,08 & 0,19 & 0,01 \\
\hline & & ref. sup. & $0,15 \mathrm{a}$ & 0,03 & 19,87 & 0,09 & 0,23 & 0,01 \\
\hline \multirow{4}{*}{$25-12-2004$} & \multirow{2}{*}{ NDVI } & ref. apar. & $0,63 \mathrm{~b}$ & 0,04 & 6,32 & 0,55 & 0,70 & $0,07 *$ \\
\hline & & ref. sup. & $0,67 \mathrm{a}$ & 0,04 & 5,89 & 0,59 & 0,78 & $0,16^{*}$ \\
\hline & \multirow{2}{*}{ GVI } & ref. apar. & $0,20 \mathrm{~b}$ & 0,02 & 9,60 & 0,17 & 0,25 & $0,17 *$ \\
\hline & & ref. sup. & $0,23 \mathrm{a}$ & 0,02 & 8,46 & 0,19 & 0,28 & $0,15^{*}$ \\
\hline \multirow{4}{*}{$26-1-2005$} & \multirow{2}{*}{ NDVI } & ref. apar. & $0,75 \mathrm{~b}$ & 0,04 & 5,36 & 0,66 & 0,81 & $0,08 *$ \\
\hline & & ref. sup. & $0,79 \mathrm{a}$ & 0,04 & 5,01 & 0,69 & 0,88 & $0,11 *$ \\
\hline & \multirow{2}{*}{ GVI } & ref. apar. & $0,27 \mathrm{~b}$ & 0,03 & 10,72 & 0,22 & 0,32 & $0,33 *$ \\
\hline & & ref. sup. & $0,33 \mathrm{a}$ & 0,03 & 8,96 & 0,25 & 0,37 & $0,08^{*}$ \\
\hline \multirow{4}{*}{$11-2-2005$} & \multirow{2}{*}{ NDVI } & ref. apar. & $0,72 \mathrm{~b}$ & 0,05 & 6,92 & 0,58 & 0,80 & $0,08^{*}$ \\
\hline & & ref. sup. & $0,78 \mathrm{a}$ & 0,05 & 6,38 & 0,66 & 0,86 & $0,12 *$ \\
\hline & \multirow{2}{*}{ GVI } & ref. apar. & $0,24 \mathrm{~b}$ & 0,03 & 12,34 & 0,18 & 0,29 & $0,34 *$ \\
\hline & & ref. sup. & $0,29 \mathrm{a}$ & 0,03 & 10,16 & 0,23 & 0,34 & $0,38 *$ \\
\hline
\end{tabular}

ref. apar.: imagens sem correção atmosférica; ref. sup.: imagens com correção atmosférica.

Para cada índice de vegetação e em cada data das imagens, médias seguidas de mesma letra não diferem significativamente entre si, segundo teste de t, com nível de $5 \%$ de probabilidade.

* possuem características da distribuição de normalidade, segundo Anderson-Darling, com nível de 5\% de significância. 
Observa-se que os dados de reflectância apresentaram a mesma tendência temporal, havendo um acréscimo gradual entre as datas de 23-11-2004 a 26-1-2005 com posterior decréscimo na data de 11-2-2005, característico da fase de senescência. Mostram ainda que, na maioria das datas, as médias dos índices apresentaram-se diferentes, mostrando a importância da correção atmosférica, e as implicações disto serão discutidas mais adiante.

Os coeficientes de variação (C.V.) foram mais altos quando comparados aos valores sugeridos por GOMES (2000). Isso, provavelmente, ocorreu pela grande diversidade existente nas datas de plantio das áreas com a cultura de soja, dentro do mesmo município, acarretando diferentes períodos de desenvolvimento da cultura entre as áreas. Assim mesmo, os resultados apresentaram-se sempre abaixo de $20 \%$, sendo que $45 \%$ dos índices têm alta homogeneidade, $50 \%$ têm média homogeneidade e 5\% alta heterogeneidade. Não apresentaram normalidade as variáveis de NDVI e GVI ref. apar. nas datas de 23-11-2004 e 9-12-2004, bem como NDVI e GVI ref. sup. de 9-122004. Os valores mínimos de NDVI $(0,23)$ e GVI $(0,03)$ foram encontrados para o fator de reflectância aparente na data de 23-11-2004, e os valores máximos NDVI $(0,88)$ e GVI $(0,37)$ encontrados para o Ref. sup. na data de 26-1-2005. No nível municipal, foram realizadas análises da relação existente entre os dados espectrais e a produtividade da soja, utilizando dados dos sensores Landsat 5/TM (NDVI e GVI) e dados da produtividade oficial (SEAB/DERAL, 2005) dos 36 municípios.

Na Tabela 3, apresenta-se a matriz de correlação linear de Pearson e a matriz de correlação não paramétrica de Sperman, obtidas entre os parâmetros do NDVI de Ref. apar. e Ref. sup., em cada data das imagens e para a imagem média de todas as datas, com os dados de produtividade oficial dos municípios, para a safra de 2004/2005. Observa-se que as correlações maiores ocorrem para a imagem média, com $r=0,69$ (Pearson) e $r_{s}=0,68$ (Sperman) para o NDVI de Ref. apar. com a produtividade e $\mathrm{r}=0,75$ (Pearson) e $\mathrm{r}_{\mathrm{s}}=0,72$ (Sperman) para o NDVI de Ref. sup. com a produtividade, todas significativas a 5\% de probabilidade, exceto na data de 25-12-2004 que não teve correlação significativa. Destaca-se que não houve grandes diferenças entre as correlações lineares de Pearson e as correlações não paramétricas de Sperman nos parâmetros em estudo.

TABELA 3. Correlações obtidas entre os parâmetros do NDVI e a produtividade municipal, na safra de 2004/2005. Correlations obtained between parameters of NDVI and the municipal productivity in 2004/2005 harvest.

\begin{tabular}{ccccccccccc}
\hline \multirow{2}{*}{ Data da Imagem } & \multirow{2}{*}{$\mathrm{n}$} & \multicolumn{3}{c}{ NDVI_ref. apar. x Produtividade } & \multicolumn{3}{c}{ NDVI_ref. sup. x Produtividade } \\
\cline { 3 - 10 } & & \multicolumn{2}{c}{ Pearson } & \multicolumn{2}{c}{ Sperman } & \multicolumn{2}{c}{ Pearson } & \multicolumn{2}{c}{ Sperman } \\
\hline $23-11-2004$ & 36 & 0,68 & 0,00 & 0,69 & 0,00 & 0,74 & 0,00 & 0,73 & 0,00 \\
$9-12-2004$ & 36 & 0,49 & 0,00 & 0,47 & 0,03 & 0,51 & 0,00 & 0,48 & 0,00 \\
$25-12-2004$ & 36 & 0,06 & $0,73 \mathrm{~ns}$ & 0,03 & $0,85 \mathrm{~ns}$ & 0,12 & $0,49 \mathrm{~ns}$ & 0,11 & $0,49 \mathrm{~ns}$ \\
$26-1-2005$ & 36 & 0,42 & 0,01 & 0,45 & 0,05 & 0,58 & 0,00 & 0,60 & 0,00 \\
$11-2-2005$ & 36 & $-0,40$ & 0,01 & $-0,38$ & 0,17 & $-0,39$ & 0,02 & $-0,41$ & 0,01 \\
\hline Médio & 36 & $\underline{0,69}$ & 0,00 & $\underline{0,68}$ & 0,00 & $\underline{0,75}$ & 0,00 & $\underline{0,72}$ & 0,00 \\
\hline
\end{tabular}

n - amostras utilizadas; $\mathrm{r}$ - coeficiente de correlação linear de Pearson; ns - correlação não significativa com 5\% de probabilidade; ref. apar. - imagens sem correção atmosférica; ref. sup. - imagens com correção atmosférica.

$\mathrm{Na}$ Tabela 4, apresentam-se os modelos utilizando regressão linear simples, obtidos entre o NDVI (aparente e de superfície) e os dados de produtividade oficial dos municípios. Nota-se que os coeficientes de determinação apresentados pelas imagens médias de todas as datas foram de $\mathrm{R}^{2}=56,25 \%$ para o NDVI de superfície e $\mathrm{R}^{2}=47,61 \%$ para o NDVI aparente. Desse modo, de $47 \%$ a $59 \%$ da variabilidade da produtividade da soja, nos municípios, podem ser explicados pelos modelos através do NDVI aparente e de superfície provenientes de todo o ciclo de desenvolvimento da soja, respectivamente. 
TABELA 4. Modelos utilizando regressão linear simples obtida entre os parâmetros do NDVI e a produtividade municipal, na safra de 2004/2005. Models using simple linear regression obtained between the parameters of NDVI and the municipal productivity in the $2004 / 2005$ harvest.

\begin{tabular}{|c|c|c|c|}
\hline \multirow{2}{*}{ Data da Imagem } & \multirow[b]{2}{*}{$\mathrm{n}$} & \multicolumn{2}{|c|}{ NDVI } \\
\hline & & Modelo & Coeficiente de Determinação (\%) \\
\hline \multirow[b]{2}{*}{$23-11-2004$} & \multirow[b]{2}{*}{36} & $\mathrm{P}=0,9573+5,157$ NDVI_ref.. apar. & $\mathrm{R}^{2}=46,24$ \\
\hline & & $\mathrm{P}=0,7812+5,009$ NDVI_ref. sup. & $\mathrm{R}^{2}=54,76$ \\
\hline \multirow{2}{*}{$9-12-2004$} & \multirow{2}{*}{36} & $\mathrm{P}=1,581+2,131$ NDVI_ref. apar. & $\mathrm{R}^{2}=24,01$ \\
\hline & & $\mathrm{P}=1,582+1,919$ NDVI_ref. sup. & $\mathrm{R}^{2}=26,01$ \\
\hline \multirow{2}{*}{$25-12-2004$} & \multirow{2}{*}{36} & $\mathrm{P}=2,219+0,468$ NDVI_ref. apar. & $\mathrm{R}^{2}=0,36$ \\
\hline & & $\mathrm{P}=1,915+0,885$ NDVI_ref. sup. & $\mathrm{R}^{2}=1,44$ \\
\hline \multirow[b]{2}{*}{$26-1-2005$} & \multirow[b]{2}{*}{36} & $\mathrm{P}=0,3402+2,914$ NDVI_ref. apar. & $\mathrm{R}^{2}=17,70$ \\
\hline & & $P=-0,4088+3,664$ NDVI_ref. sup. & $\mathrm{R}^{2}=33,60$ \\
\hline \multirow[b]{2}{*}{$11-2-2005$} & \multirow[b]{2}{*}{36} & $\mathrm{P}=4,292$ - 2,460 NDVI_ref. apar. & $\mathrm{R}^{2}=16,00$ \\
\hline & & $\mathrm{P}=4,283-2,256$ NDVI_ref. sup. & $\mathrm{R}^{2}=15,21$ \\
\hline \multirow{2}{*}{ Médio } & \multirow[b]{2}{*}{36} & $P=-4,284+11,96$ NDVI_ref. apar. & $\mathrm{R}^{2}=47,61$ \\
\hline & & $P=-3,719+10,08$ NDVI_ref. sup. & $\mathrm{R}^{2}=56,25$ \\
\hline
\end{tabular}

$\mathrm{n}$ - número de amostras utilizadas nas regressões; P - produtividade da soja; *resíduos não apresentam distribuição normal com nível de 5\% de significância; ref. apar. - imagens sem correção atmosférica; ref. sup. - imagens com correção atmosférica.

Para os dados de NDVI municipal, da safra de 2004/2005, não foi possível perceber a sua saturação. As causas, provavelmente, foram as diferentes datas de plantio e variedades de soja utilizadas nas áreas cultivadas.

Uma observação pertinente é sobre os valores muito baixos de coeficiente de determinação do NDVI para a data de 25-12-2004 (0,36 e 1,44), ocorrido provavelmente por a imagem apresentar contaminação por substratos de nuvens ("haze"), e esses não foram possíveis remover pela metodologia utilizada para a retirada dos pixels contaminados com nuvens, podendo assim ter alterado os valores reais do NDVI nesta imagem. A Tabela 5 apresenta a matriz de correlação linear de Pearson e a matriz de correlação não paramétrica de Sperman entre os parâmetros do GVI de Ref. apar. e de Ref. sup., em cada uma das datas das imagens, assim como com a imagem média de todas as datas versus os dados de produtividade oficial.

TABELA 5. Correlações obtidas entre os parâmetros do GVI e a produtividade municipal, na safra de 2004/2005. Correlations obtained between the parameters from GVI and the municipal productivity in the $2004 / 2005$ harvest.

\begin{tabular}{cccccccccc}
\hline \multirow{2}{*}{ Data da Imagem } & \multirow{2}{*}{$\mathrm{n}$} & \multicolumn{3}{c}{ GVI_ref. apar. $x$ Produtividade } & \multicolumn{3}{c}{ GVI_ref. sup. x Produtividade } \\
\cline { 3 - 10 } & & \multicolumn{2}{c}{ Pearson } & \multicolumn{2}{c}{ Sperman } & \multicolumn{2}{c}{ Pearson } & \multicolumn{2}{c}{ Sperman } \\
\hline $23-11-2004$ & 36 & 0,64 & 0,00 & 0,62 & 0,00 & 0,64 & 0,00 & 0,62 & 0,00 \\
$9-12-2004$ & 36 & 0,42 & 0,00 & 0,38 & 0,02 & 0,55 & 0,00 & 0,52 & 0,00 \\
$25-12-2004$ & 36 & 0,43 & 0,00 & 0,44 & 0,01 & 0,51 & 0,00 & 0,46 & 0,01 \\
$26-1-2005$ & 36 & 0,61 & 0,00 & 0,58 & 0,00 & 0,64 & 0,00 & 0,61 & 0,00 \\
$11-2-2005$ & 36 & $-0,37$ & 0,02 & $-0,37$ & 0,03 & $-0,38$ & 0,02 & $-0,40$ & 0,01 \\
\hline Médio & 36 & $\underline{0,75}$ & 0,00 & $\underline{0,74}$ & 0,00 & $\underline{0,87}$ & 0,00 & $\underline{0,85}$ & 0,00 \\
\hline
\end{tabular}

n - amostras utilizadas; $r$ - coeficiente de correlação linear de Pearson; ref. apar. - imagens sem correção atmosférica; ref. sup. imagens com correção atmosférica.

Os dados de correlação mais altos para o índice GVI foram obtidos com a imagem média, em que $r=0,75$ (Pearson) e $r_{s}=0,74$ (Sperman) para o GVI de Ref. apar. com produtividade, e $r=0,87$ (Pearson) e $\mathrm{r}_{\mathrm{s}}=0,85$ (Sperman) para o GVI de Ref. sup. com produtividade, todos significativos com $5 \%$ de probabilidade. Assim como para o índice NDVI, para os dados de GVI também não 
houve grandes diferenças entre as correlações lineares de Pearson e as correlações não paramétricas de Sperman.

Os modelos de regressão linear simples, apresentados na Tabela 6, são referentes aos mesmos parâmetros do GVI utilizados na correlação versus os dados de produtividade oficial de cada município. Os resultados mostram que as imagens médias apresentaram os melhores coeficientes de determinação, sendo que os valores foram de $\mathrm{R}^{2}=76,2 \%$ para o GVI de superfície e $57,4 \%$ para o GVI aparente. Para o GVI da safra de 2004/2005, pode-se notar que o GVI de superfície mais explicou a variabilidade da produtividade da soja na região abrangida pelos 36 municípios, com $76 \%$, aproximadamente, de explicação. Para esta safra, ficou evidenciado que o índice GVI com as imagens médias de todas as datas, foi a que alcançou os maiores valores de $\mathrm{R}^{2}$ e não o índice NDVI. Ainda assim, percebem-se diferenças nas regressões para os índices NDVI e GVI quanto às refletâncias aparente e de superfície.

TABELA 6. Modelos utilizando regressão linear simples obtida entre os parâmetros do GVI e a produtividade municipal, na safra de 2004/2005. Models using simple linear regression obtained between the parameters of GVI and the municipal productivity in the $2004 / 2005$ harvest.

\begin{tabular}{|c|c|c|c|}
\hline \multirow{2}{*}{ Data da Imagem } & \multirow{2}{*}{$\mathrm{n}$} & \multicolumn{2}{|c|}{ GVI } \\
\hline & & Modelo & Coeficiente de Determinação (\%) \\
\hline \multirow{2}{*}{$23-11-2004$} & \multirow[b]{2}{*}{36} & $\mathrm{P}=1,802+13,29$ GVI_ref. apar. & $\mathrm{R}^{2}=41,2$ \\
\hline & & $\mathrm{P}=1,602+11,58$ GVI_ref. sup. & $\mathrm{R}^{2}=41,9$ \\
\hline \multirow{2}{*}{$9-12-2004$} & \multirow{2}{*}{36} & $\mathrm{P}=1,905+5,117$ GVI_ref. apar. & $\mathrm{R}^{2}=18,0$ \\
\hline & & $\mathrm{P}=1,635+5,829$ GVI_ref. sup. & $\mathrm{R}^{2}=30,6$ \\
\hline \multirow{2}{*}{ 25-12-2004 } & \multirow{2}{*}{36} & $\mathrm{P}=1,319+5,74$ GVI_ref. apar. & $\mathrm{R}^{2}=18,8$ \\
\hline & & $P=0,9379+6,675 G V I \_r e f$. sup. & $\mathrm{R}^{2}=26,1$ \\
\hline \multirow{2}{*}{$26-1-2005$} & \multirow{2}{*}{36} & $\mathrm{P}=0,4472+7,38$ GVI_ref. apar. & $R^{2}=37,6$ \\
\hline & & $P=0,3577+6,445$ GVI_ref. sup. & $\mathrm{R}^{2}=42,0$ \\
\hline \multirow{2}{*}{$11-2-2005$} & \multirow{2}{*}{36} & $\mathrm{P}=3,624$ - 4,557 GVI_ref. apar. & $\mathrm{R}^{2}=14,4$ \\
\hline & & $P=3,719$ - 4,075 GVI_ref. sup. & $\mathrm{R}^{2}=14,6$ \\
\hline \multirow{2}{*}{ Médio } & \multirow{2}{*}{36} & $\mathrm{P}=-1,870+24,24$ GVI_ref. apar. & $\mathrm{R}^{2}=57,4$ \\
\hline & & $P=-2,794+24,21$ GVI_ref. sup. & $\mathrm{R}^{2}=76,2$ \\
\hline
\end{tabular}

$\mathrm{n}$ - número de amostras utilizadas nas regressões; P - produtividade da soja; *resíduos não apresentam distribuição normal com nível de 5\% de significância; - ref. apar. - imagens sem correção atmosférica; ref. sup. - imagens com correção atmosférica.

Seguindo as análises efetuadas, na Tabela 7, encontram-se os modelos de regressão linear múltipla, ajustados entre os dados de NDVI de fator de refletância aparente e de superfície, com dados de produtividade oficial dos municípios, para a safra de 2004/2005.

Para esses modelos, foram obtidos os coeficientes de determinação $\mathrm{R}^{2}=53,6 \%$ para o NDVI aparente e $\mathrm{R}^{2}=64,0 \%$ para o NDVI de superfície, levando em consideração todas as datas das imagens. Ao se implantar o método de Stepwise de melhor escolha do modelo, no ajuste dos modelos, os resultados obtidos foram de $\mathrm{R}^{2}=46,3 \%$ para os dados de refletância aparente com a imagem na data de 23-11-2004 significativa. Já para os dados de refletância de superfície, as imagens significativas foram 23-11-2004 e 26-1-2005, com $\mathrm{R}^{2}=57,0 \%$. Desse modo, os resultados indicam que os modelos foram capazes de explicar $46,3 \%$ e $57 \%$, respectivamente, da variabilidade encontrada na produtividade da cultura da soja. 
TABELA 7. Modelos utilizando regressões lineares múltiplas, segundo todas as imagens, entre os parâmetros do NDVI e a produtividade municipal, na safra de 2004/2005. Models using multiple linear regressions according to all the parameters of the images between NDVI and the municipal productivity in the $2004 / 2005$ harvest.

\begin{tabular}{|c|c|c|}
\hline \multirow{2}{*}{$\begin{array}{l}\text { Tratamento de } \\
\text { Refletância }\end{array}$} & \multicolumn{2}{|l|}{ NDVI } \\
\hline & Modelo & Coeficiente de Determinação (\%) \\
\hline \multirow[t]{2}{*}{ ref. apar. } & $\begin{array}{l}\mathrm{P}=-2,31+4,74 \text { NDVI } 23-11-2004+0,65 \text { NDVI } \\
9-12-2004+1,66 \text { NDVI } 25-12-2004+1,69 \text { NDVI } \\
26-1-2005+1,11 \text { NDVI } 11-2-2005\end{array}$ & $\mathrm{R}^{2}=53,6^{\text {completa }}$ \\
\hline & $\mathrm{P}=0,96+5,16$ NDVI $23-11-2004$ & $\mathrm{R}^{2}=46,3^{\text {Stepwise }}$ \\
\hline \multirow[t]{2}{*}{ ref. sup. } & $\begin{array}{l}\mathrm{P}=-2,59+4,21 \text { NDVI } 23-11-2004+0,453 \mathrm{NDVI} \\
9-12-2004+1,12 \text { NDVI } 25-12-2004+2,10 \text { NDVI } \\
26-1-2005+1,27 \text { NDVI } 11-2-2005\end{array}$ & $\mathrm{R}^{2}=64,0^{\text {completa }}$ \\
\hline & $\begin{array}{l}\mathrm{P}=-0,20+4,09 \text { NDVI } 23-11-2004+1,63 \mathrm{NDVI} \\
26-1-2005\end{array}$ & $\mathrm{R}^{2}=57,0^{\text {Stepwise }}$ \\
\hline
\end{tabular}

Na Tabela 8, estão os modelos ajustados utilizando a regressão linear múltipla entre os dados do GVI de refletâncias aparente e de superfície, com dados de produtividade oficial dos municípios, para a safra de 2004/2005. Os coeficientes de determinação obtidos com a utilização de todas as variáveis foram: $\mathrm{R}^{2}=67,4 \%$ para o GVI aparente e $\mathrm{R}^{2}=80,2 \%$ para o GVI de superfície.

Quando se utilizou o método de melhor escolha de modelos Stepwise no ajuste dos modelos, foram obtidos resultados diferentes somente para os dados de refletância aparente, onde a imagem da data de 9-12-2004 não se mostrou significativa e, consequentemente, foi retirada do modelo, apresentando, assim, um $\mathrm{R}^{2}=67,3 \%$. Para os dados do GVI de refletância de superfície, o método de Stepwise classificou todas as imagens como significativas, não alterando os coeficientes de determinação $\mathrm{R}^{2}$ encontrados.

TABELA 8. Modelos utilizando regressões lineares múltiplas, segundo todas as imagens entre os parâmetros do GVI e a produtividade municipal, na safra de 2004/2005 Models using multiple linear regressions according to all the images between the parameters of GVI and the municipal productivity in the $2004 / 2005$ harvest.

\begin{tabular}{|c|c|c|}
\hline \multirow{2}{*}{$\begin{array}{l}\text { Tratamento de } \\
\text { Refletância }\end{array}$} & \multicolumn{2}{|l|}{ GVI } \\
\hline & Modelo & $\begin{array}{c}\text { Coeficiente de } \\
\text { Determinação }(\%)\end{array}$ \\
\hline \multirow{2}{*}{ ref. apar. } & $\begin{array}{l}\mathrm{P}=-1,33+11,4 \text { GVI } 23-11-2004+0,35 \text { GVI 9-12-2004 + 3,84 } \\
\text { GVI 25-12-2004 + 5,63 GVI 26-1-2005 + 3,33 GVI 11-2-2005 }\end{array}$ & $\mathrm{R}^{2}=67,4^{\text {completa }}$ \\
\hline & $\begin{array}{l}\mathrm{P}=-1,25+11,6 \text { GVI } 23-11-2004+3,9 \text { GVI 25-12-2004 + 5,6 } \\
\text { GVI 26-1-2005 + 3,2 GVI 11-2-2005 }\end{array}$ & $\mathrm{R}^{2}=67,3^{\text {Stepwise }}$ \\
\hline \multirow{2}{*}{ ref. sup. } & $\begin{array}{l}\mathrm{P}=-3,07+9,13 \text { GVI } 23-11-2004+2,40 \text { GVI 9-12-2004 + 5,04 } \\
\text { GVI 25-12-2004 + 5,19 GVI 26-1-2005 + 5,33 GVI 11-2-2005 }\end{array}$ & $\mathrm{R}^{2}=80,2^{\text {completa }}$ \\
\hline & $\begin{array}{l}\mathrm{P}=-3,07+9,13 \text { GVI } 23-11-2004+2,40 \text { GVI 9-12-2004 + 5,04 } \\
\text { GVI 25-12-2004 + 5,19 GVI 26-1-2005 + 5,33 GVI 11-2-2005 }\end{array}$ & $\mathrm{R}^{2}=80,2^{\text {Stepwise }}$ \\
\hline
\end{tabular}

$\mathrm{P}$ - produtividade da soja; ${ }^{\text {Stepwise }}$ - modelo ajustado com variáveis significativas, segundo o método de Stepwise; ${ }^{\text {completa }}$ - modelo ajustado utilizando todas as variáveis em estudo; ref. apar. - imagens sem correção atmosférica; ref. sup. - imagens com correção atmosférica.

Dessa maneira, o modelo para o GVI de ref. sup. foi o que mais explicou a variabilidade da produtividade da cultura da soja, nos 36 municípios estudados para safra de 2004/2005, com 80,2\% de explicação, aproximadamente. Outra informação que fica evidente nos modelos de regressão 
linear múltiplas, foi a de que os dados do índice GVI apresentaram todas as variáveis significativas, alcançando também os maiores $\mathrm{R}^{2}$ quando comparados aos dados do índice NDVI.

Na Tabela 9, apresentam-se os modelos para os dados do NDVI e do GVI de ref. apar. e ref. sup., respectivamente. Os modelos definidos com todas as variáveis apresentam coeficientes de determinação entre $\mathrm{R}^{2}=78,7 \%$ para regressão com dados de ref. apar. a $\mathrm{R}^{2}=86,7 \%$ para a regressão com dados de ref. sup. Pelo método de Stepwise, utilizado no melhor ajuste dos modelos, os resultados foram de $\mathrm{R}^{2}=76,2 \%$ para os dados de ref. apar. com as imagens significativas nas datas de 26-1-2005 e 11-2-2005 para o NDVI, e nas datas de 23-11-2004, 25-12-2004, 26-1-2005 e 11-22005 para o GVI. Para os dados de ref. sup. as imagens significativas foram de 23-11-2004, 9-122004, 25-12-2004 e 11-2-2005 para o NDVI, e para o GVI as datas de 9-12-2004 e 26-1-2005, apresentando $\mathrm{R}^{2}=84,1 \%$.

Com $84 \%$ de explicação da variabilidade da produtividade da cultura da soja, nos 36 municípios estudados, para safra de 2004/2005, o maior valor de $\mathrm{R}^{2}$ apresentado pelas variáveis dos índices NDVI e GVI de ref. sup. Com isso, percebe-se que a utilização dos índices de vegetação NDVI e GVI, conjuntamente, para a safra de 2004/2005, apresentou maiores $\mathrm{R}^{2}$ do que quando se utilizam os índices separadamente.

TABELA 9. Modelos utilizando regressões lineares múltiplas obtidas com todas as datas das imagens entre os dois parâmetros NDVI e GVI juntos versus a produtividade municipal na safra de 2004/2005. Models using multiple linear regressions obtained with all the dates of the images between the two parameters NDVI and GVI together versus the municipal productivity in the 2004/2005 harvest.

\begin{tabular}{|c|c|c|}
\hline \multirow{2}{*}{$\begin{array}{l}\text { Tratamento de } \\
\text { refletância }\end{array}$} & \multicolumn{2}{|l|}{ NDVI e GVI } \\
\hline & Modelo & $\begin{array}{c}\text { Coeficiente de } \\
\text { determinação }(\%)\end{array}$ \\
\hline \multirow[t]{2}{*}{ ref. apar. } & $\begin{array}{l}\mathrm{P}=-4,33+1,43 \text { NDVI } 23-11-2004+1,28 \text { NDVI } \\
9-12-2004+1,81 \text { NDVI } 25-12-2004-2,80 \text { NDVI } \\
26-1-2005+7,55 \text { NDVI } 11-2-2005+10,5 \text { GVI } \\
23-11-2004-1,44 \text { GVI 9-12-2004 + 1,49 GVI 25-12-2004 } \\
+11,0 \text { GVI 26-1-2005 - 10,0 GVI 11-2-2005 } \\
\end{array}$ & $\mathrm{R}^{2}=78,7^{\text {completa }}$ \\
\hline & $\begin{array}{l}\mathrm{P}=-2,42-3,03 \text { NDVI } 26-1-2005+5,91 \text { NDVI } \\
11-2-2005+14,3 \text { GVI 23-11-2004 + 4,25 GVI 25-12-2004 + } \\
10,8 \text { GVI 26-1-2005 - 7,19 GVI 11-2-2005 }\end{array}$ & $\mathrm{R}^{2}=76,2^{\text {Stepwise }}$ \\
\hline \multirow[t]{2}{*}{ ref. sup. } & $\begin{array}{l}\mathrm{P}=-4,71+2,32 \text { NDVI } 23-11-2004-1,22 \text { NDVI } \\
9-12-2004+1,47 \text { NDVI } 25-12-2004-1,52 \text { NDVI } \\
26-1-2005+3,59 \text { NDVI } 11-2-2005+6,24 \text { GVI } \\
23-11-2004+5,40 \text { GVI } 9-12-2004+2,20 \text { GVI } 25-12-2004+ \\
8,18 \text { GVI } 26-1-2005-0,50 \text { GVI } 11-2-2005\end{array}$ & $\mathrm{R}^{2}=86,7^{\text {completa }}$ \\
\hline & $\begin{array}{l}\mathrm{P}=-5,34+4,22 \text { NDVI } 23-11-2004-1,76 \text { NDVI } \\
9-12-2004+1,72 \text { NDVI } 25-12-2004+3,49 \text { NDVI } \\
11-2-2005+8,09 \text { GVI 9-12-2004 + 6,35 GVI 26-1-2005 }\end{array}$ & $\mathrm{R}^{2}=84,1^{\text {Stepwise }}$ \\
\hline
\end{tabular}

Como observado anteriormente, todas as análises das correlações e regressões dos índices de vegetação versus a produtividade apresentaram a mesma tendência, com melhores resultados, quando considerado todo o perfil espectral do ciclo, indicando que o monitoramento do perfil espectral temporal, traduzido pelos índices NDVI e GVI nos 36 municípios, pôde explicar grande parte da variabilidade da produtividade da soja nas duas safras. Resultados semelhantes, considerando todo o ciclo de desenvolvimento da cultura, foram encontrados por BENVENUTI (2005), trabalhando com índices das imagens do Landsat $7 / \mathrm{ETM}^{+}$para a cana-de- 
-açúcar e por LIU \& KOGAN (2002) no monitoramento da soja, em várias regiões do Brasil, utilizando índices de vegetação derivados das imagens Landsat 7/ETM ${ }^{+}$e MODIS.

O melhor resultado obtido nas regressões lineares simples com as imagens médias para o índice NDVI do Landsat 5/TM foi de 56,25\% de explicação da variabilidade da produtividade da soja na região estudada, para a safra de 2004/2005. Para o índice GVI do Landsat 5/TM, o melhor resultado foi obtido com a imagem média da safra de 2004/2005, com 76,2\% de explicação da variabilidade da produtividade.

Comparando as regressões lineares múltiplas realizadas, os resultados obtidos foram melhores do que as regressões lineares simples. Esse foi o melhor resultado obtido de todas as análises efetuadas entre dados espectrais e produtividade. Para o índice GVI do Landsat 5/TM (método Stepwise), o melhor resultado foi de $\mathbf{8 0 , 2 \%}$ para ref. sup.

As correlações mais altas obtidas nas duas safras estudadas para o índice NDVI estão próximas das obtidas por vários autores que também trabalharam com o NDVI em relações com a produtividade das culturas, tais como, MA et al. (2001) que obtiveram coeficiente de determinação de $80 \%$ para a soja, e WOOD et al. (2003), que obtiveram coeficiente de determinação de $70 \%$ para o trigo. THENKABAIL et al. (2000) e ZHA et al. (2003) também trabalharam com o índice NDVI, entretanto foi para determinação de variáveis biofísicas de plantas e não somente para produtividade. Nessas pesquisas, os autores obtiveram coeficientes de determinação próximos aos encontrados nesta pesquisa.

Para os dados do índice de vegetação GVI, os melhores resultados encontrados corroboram, ou estão muito próximos dos resultados encontrados por MACHADO (2003) e BENVENUTI (2005), com $\mathrm{R}^{2}=83 \%$, e FORTES (2003), com $\mathrm{R}^{2}=69,85 \%$, mesmo todos esses autores trabalhando com a cultura da cana-de-açúcar e não com a cultura da soja.

Em uma análise mais detalhada, não foi encontrada a existência de multicolinearidade entre os índices utilizados conjuntamente (NDVI e GVI). Nos cálculos para avaliar a Autocorrelação serial, implementada por meio do teste de Durbin-Watson, não foi encontrada autocorrelação serial para os modelos ajustados pelo método de Stepwise, para fator de refletância de superfície $(\mathrm{d}=2,11)$ e normalizada $(\mathrm{d}=2,14)$. Entretanto, o teste não foi conclusivo para os dados de ref. apar. $(\mathrm{d}=2,59)$.

\section{Diagnósticos de modelos com os dados Landsat 5/TM}

Na safra estudada, os modelos que apresentaram os melhores resultados de regressão linear foram os de ref. sup. para o GVI e para o modelo de regressão linear múltipla com os dados de NDVI e GVI, conjuntamente.

Para o modelo ajustado com o GVI ref. sup., foram os municípios de Iguatú e o de Quarto Centenário que se apresentaram como pontos discrepantes, mas não influentes do ajuste do modelo. Apresentaram-se como pontos de grande influência, no ajuste do modelo (pontos discrepantes no espaço dos preditores - $\mathrm{DM}_{\mathrm{i}}$ ), os municípios Araruna e Palotina. E demonstraram que a retirada motiva grande alteração no ajuste do modelo (ponto Leverage - $\mathrm{h}_{\mathrm{ii}}$ ), devendo os mesmos serem examinados com algum cuidado, os municípios de Araruna, Palotina e Tuneiras.

Da mesma maneira, os dados do índice GVI fator de refletância superfície também apresentou o melhor ajuste do modelo linear múltiplo. Para este modelo, três municípios: Cascavel, Iguatú e Quarto Centenário foram considerados supostos valores discrepantes pelas análises de resíduos $\mathrm{r}_{\mathrm{i}}, \mathrm{z}_{\mathrm{i}}$ e $t_{i}$. Sendo que apenas os municípios de Anahy e Campo Bonito se apresentaram como pontos discrepantes no espaço dos preditores, ou seja, pontos influentes no ajuste do modelo $\left(\mathrm{DM}_{\mathrm{i}}\right)$. Ao mesmo tempo, a retirada de um deles motivaria uma grande alteração no ajuste desse modelo (ponto Leverage $-\mathrm{h}_{\mathrm{ii}}$ ).

Por seguimento, no modelo linear múltiplo ajustado com os dados dos índices NDVI e GVI de ref. sup., conjuntamente, apenas o município de Cascavel foi considerado um valor discrepante 
pelas análises de resíduos $\mathrm{r}_{\mathrm{i}}, \mathrm{z}_{\mathrm{i}}$ e $\mathrm{t}_{\mathrm{i}}$, não tendo, para este modelo, nenhum município com características de ponto influente no ajuste do modelo (ponto discrepante no espaço dos preditores $\left.\mathrm{DM}_{\mathrm{i}}\right)$.

Por meio das análises realizadas, utilizando a distância de Cook $\left(\mathrm{D}_{\mathrm{i}}\right)$ e da distância Welsch-Kuh (DFFITS) não foram encontrados pontos influentes nos modelos de regressão efetuados com os dados municipais de NDVI e GVI e nos valores por estes preditos, respectivamente. Percebe-se, por meio dos testes de diagnósticos realizados para os dados municipais do Landsat 5/TM, que, para os modelos lineares simples, a maior parte dos municípios que se apresentava como pontos influentes para o índice NDVI, apresentava-se influente, também, para o índice GVI.

\section{CONCLUSÕES}

- O trabalho mostra a importância, no caso do estudo de previsão da safra de soja, de se considerar o ciclo da cultura como um todo ao se constatar que a média das imagens proporciona melhores resultados.

- A utilização de regressões múltiplas, levando em conta os dois índices em todas as datas, propiciou melhores modelos de previsão da produtividade da soja.

- Técnicas de diagnósticos mostraram que são ferramentas essenciais na análise de pontos discrepantes quando a análise se dá em macrorregiões com alta variabilidade dos dados.

- Em análises de macrorregiões, modelos espectrais funcionam bem em termos de custo e benefício, isto é, imagens de médias resoluções, como as do Landsat 5/TM, as quais agora são disponibilizadas gratuitamente, tornam-se ferramentas extremamente poderosas na medida em que geram os modelos espectrais.

\section{REFERÊNCIAS}

ALMEIDA, T.I.R.; SOUZA FILHO, C.R. de; ROSSETTO, R. ASTER and Landsat ETM + images applied to sugarcane yield forecast. International Journal of Remote Sensing, London, v.27, p.4.057-4.069, 2006.

BENVENUTI, F.A. Relação de índices espectrais de vegetação com a produtividade da cana-de- açúcar e atributos edáficos. 2005. 115 f. Dissertação (Mestrado em Engenharia Agrícola) Faculdade de Engenharia Agrícola, Universidade Estadual de Campinas, Campinas, 2005.

BOATWRIGHT, G.O.; WHITEFIELD, V.S. Early warning and crop condition assessment research. IEEE Transactions on Geoscienses and Remote Sensing, New York, v.24, n.1, p.56-64, 1986.

CHANDER, G.; MARKHAM, B. Revised Landsat-5 TM Radiometric Calibration Procedures and Postcalibration Dynamic Ranges. IEEE Transactions on Geoscience and Remote Sensing, New York, v.41, n.11, p.2.764-2.677, 2003.

CRÓSTA, A.P. Processamento digital de imagens de sensoriamento remoto. Campinas: UNICAMP, 1993. $170 \mathrm{p}$.

DORAISWAMY, P.C.; HATFIELD, J.L.; JACKSON, T.J.; AKHMEDOV, B.; PRUEGER, J.; STERN, A. Crop condition and yield simulations using Landsat and MODIS. Remote Sensing of Environment, New York, v.92, p.548-559, 2004.

FORTES, C. Discriminação varietal e estimativa de produtividade agroindustrial de cana-de-açúcar pelo sensor orbital ETM+/LANDSAT7. 203. 131 f. Dissertação (Mestrado em Agronomia) Escola Superior de Agricultura "Luiz de Queiroz", Universidade de São Paulo, Piracicaba, 2003.

GALEA, M.; PAULA, G.A.; URIBE-OPAZO, M. On influence diagnostic in univariate elliptical linear regression models. Statistical Papers, Heidelberg, v.44, n.1, p.23-45, 2003. 
GOMES, F.P. Curso de estatística experimental. 14.ed. Piracicaba: ESALQ, 2000. 477 p.

GROTEN, S.M.E. NDVI-crop monitoring and early yield assessment of Burkina Faso. International Journal of Remote Sensing, London, v.14, n.8, p.1.495-1.515, 1993.

IBGE. Levantamento da produção agrícola. Disponível em:

$<\mathrm{http}: / /$ www.sidra.ibge.gov.br/bda/tabela/listabl.asp?c=1618\&n=0\&z=t\&o=1\&i=P>. Acesso em: 10 out. 2008a.

IBGE. Mapas tematicos de solo. Disponível em: <http://mapas.ibge.gov.br/solos/viewer.htm>. Acesso em: 10 nov. 2008b.

KASTENS, J.H.; KASTENS, T.L.; KASTENS, D.L.A.; PRICE, K.P.; MARTINKO, E.A.; LEE, R.Y. Image masking for crop yield forecasting using AVHRR NDVI time series imagery. Remote Sensing of Environment, New York, v.99, p.341-356, 2005.

KAUTH, R.J.; THOMAS G.S. The tasseled cap - a graphic description of the spectral-temporal development of agricultural crops as seen by Landsat. In: SYMPOSIUM ON MACHINE PROCESSING OF REMOTELY SENSED DATA, 1976, West Lafayette. Proceedings... p.41-50.

LIU, W.T.; KOGAN, F. Monitoring brazilian soybean production using NOAA/AVHRR based vegetation condition indices. International Journal of Remote Sensing, London, v.23, n.6, p.1.1611.179, 2002.

MA, B.L.; DWYER, L.M.; COSTA, C.; COBER, E.R.; MORRISON, M.J. Early prediction of soybean yield from canopy reflectance measurements. Agronomy Journal, Madison, v.93, p.1.227$1.234,2001$.

MACHADO, H.M. Determinação da biomassa de cana-de-açúcar considerando a variação espacial de dados espectrais do satélite Landsat 7 - ETM+. 2003. 59 f. Dissertação (Mestrado em Engenharia Agrícola) - Faculdade de Engenharia Agrícola, Universidade Estadual de Campinas, 2003.

MELO, R.W.; FONTANA, D.C.; BERLATO, M.A.; DUCATI, J.R. An agrometeorologicalspectral model to estimate soybean yield, applied to southern Brazil. International Journal of Remote Sensing, v.29, n.14, p.4.013-4.028, 2008.

MERCANTE, E. Dinâmica espectral da cultura da soja ao longo do ciclo vegetativo e sua relação com a produtividade na região oeste do Paraná. 2007. 221 f. (Doutorado) - Faculdade de Engenharia Agrícola, Universidade Estadual de Campinas, Campinas, 2007.

MERCANTE, E.; ANTUNES, J.F.G.; LAMPARELLI, R.A.C.; ROCHA, J.V. Estimativa de área da cultura de soja com imagens Landsat 5/TM por meio de matriz de erros. In: CONGRESSO BRASILEIRO DE ENGENHARIA AGRÍCOLA, 35., 2006, João Pessoa. Anais... Jaboticabal: Associação Brasileira de Engenharia Agrícola, 2006. 1 CD-ROM.

MINITAB. Minitab for windows - Version Release 14. State College-PA, 2004. Statistical Software.

ROUSE, J.W.; HAAS, R.H.; SCHELL, J.A.; DEERING, D.W. Monitoring vegetation systems in the great plains with ERTS. In: EARTH RESOURCES TECHNOLOGY SATELLITE-1 SYMPOSIUM, 3., 1973, Washington. Proceedings... Washington: NASA, Goddard Space Flight Center, 1973. v.1, p.309- 317. (NASA SP-351)

RUDORFF, B.F.T.; BATISTA G.T. Yield estimation of sugarcane based on agrometeorologicalspectral models. Remote Sensing of Environment, New York, v.33, p.183-192, 1990.

SEAB/DERAL. Secretaria da Agricultura e do Abastecimento do Paraná. Departamento de Economia Rural. Disponível em: <http://www.pr.gov.br/seab> Acesso em: dez. 2005. 
SIMÕES, M.D.S.; ROCHA, J.V.; LAMPARELLI, R.A.C. Spectral variables, growth analysis and yield of sugarcane. Scientia Agricola, Piracivaba, v.62, p.199-207, 2005.

SPSS. SPSS for windows: version release 13.0: bried guide. Washington, 2001. Statistical Software.

THENKABAIL, P.S.; SMITH, R.B.; PAUW, E.D. Hyperspectral vegetation indices and their relationships with agricultural crop characteristics. Remote Sensing of Environment, New York, v.71, p.158-182, 2000.

WOOD, G.A.; TAYLOR, J.C.; RICHARD, J.G. Calibration Methodology for mapping within-field crop variability using remote sensing. Biosystems Engineering, London, v.84, n.4, p.409-423, 2003.

ZHA, Y.; GAO, J.; NI, S.; LIU, Y.; JIANG, J.; WEI, Y. A spectral reflectance-based approach to quantification of grassland cover from Landsat TM imagery. Remote Sensing of Environment, New York, v.87, p.371-375, 2003.

ZULLO JÚNIOR, J. Correção atmosférica de imagens de satélite e aplicações. 1994. 189 f. Tese (Doutorado em Engenharia Elétrica) - Faculdade de Engenharia Elétrica, Universidade Estadual de Campinas, Campinas, 1994. 